\title{
Liver Abnormalities in Patients with Psoriatic Arthritis
}

\author{
Rattapol Pakchotanon, Justine Yang Ye, Richard J. Cook, Vinod Chandran, \\ and Dafna D. Gladman (1D)
}

ABSTRACT. Objective. We aimed to determine the prevalence and incidence, and to identify the factors associated with liver abnormalities in patients with psoriatic arthritis (PsA).

Methods. From a longitudinal cohort study, we identified PsA patients with either elevated serum transaminase or alkaline phosphatase levels or liver disease after the first visit to the PsA clinic (cases). Controls were subjects from the same cohort who never had such abnormalities or liver disease. Cases and controls were matched 1:1 by sex, age at the first clinic visit, and followup duration; variables at the onset of the first appearance of liver test abnormality associated with liver abnormalities were identified using univariate logistic and multivariate logistic regression analyses. Results. Among 1061 patients followed in the PsA clinic, 343 had liver abnormalities. Two hundred fifty-six patients who developed liver abnormalities after the first visit were identified as cases, and 718 patients were identified as controls. The prevalence of liver abnormalities was $32 \%$ and the incidence was 39/1000 patient-years where there were 256 cases over 6533 total person-years in the PsA cohort. Liver abnormalities were detected after a mean (SD) followup duration of $8.3 \pm 7.8$ years. The common causes of liver abnormalities were drug-induced hepatitis and fatty liver. Independent factors associated with liver abnormalities were higher body mass index (BMI), daily alcohol intake, higher damaged joint count, elevated C-reactive protein, and use of methotrexate, leflunomide, or tumor necrosis factor inhibitors.

Conclusion. Liver abnormalities are common among patients with PsA and are associated with higher BMI, more severe disease, and certain therapies. (First Release April 15 2020; J Rheumatol 2020;47:847-53; doi:10.3899/jrheum.181312)

Key Indexing Terms:

PSORIATIC ARTHRITIS LIVER BODY MASS INDEX BIOLOGICS DMARD

From the Psoriatic Arthritis Program, Centre for Prognosis Studies in the Rheumatic Diseases, Toronto Western Hospital, University Health Network, Toronto; Department of Statistics and Actuarial Science, University of Waterloo, Waterloo; University of Toronto, Department of Medicine, Division of Rheumatology, University of Toronto, Toronto; Krembil Research Institute, Toronto Western Hospital, University Health Network, Toronto, Ontario, Canada; Rheumatic Disease Unit, Department of Internal Medicine, Phramongkutlao Hospital and College of Medicine, Bangkok, Thailand.

The Psoriatic Arthritis Program is funded in part by The Arthritis Society, Canadian Institutes of Health Research, and the Krembil Foundation.

R. Pakchotanon, MD, Centre for Prognosis Studies in the Rheumatic Diseases, Toronto Western Hospital, and Rheumatic Disease Unit, Department of Internal Medicine, Phramongkutlao Hospital and College of Medicine; J.Y. Ye, Biostatistician, Centre for Prognosis Studies in the Rheumatic Diseases, Toronto Western Hospital; R.J. Cook, PhD, Professor, Department of Statistics and Actuarial Science, University of Waterloo; V. Chandran, MBBS, MD, DM, PhD, Assistant Professor, University of Toronto, Department of Medicine, Division of Rheumatology, University of Toronto, Co-Director, Psoriatic Arthritis Program, Centre for Prognosis Studies in the Rheumatic Diseases, Toronto Western Hospital, University Health Network; D.D. Gladman, MD, FRCPC, Director, Psoriatic Arthritis Program, Centre for Prognosis Studies in the Rheumatic Diseases, Senior Scientist, Krembil Research Institute, Toronto Western Hospital, University Health Network.

Address correspondence to Dr. D.D. Gladman, Psoriatic Arthritis

Program, Centre for Prognosis Studies in the Rheumatic Diseases, Krembil Research Institute, University Health Network,

399 Bathurst St., 1E410B, Toronto, Ontario M5T 2S8, Canada.

E-mail:Dafna.gladman@utoronto.ca

Accepted for publication September 24, 2019.
Psoriatic arthritis (PsA) is a chronic inflammatory disease that affects the skin and musculoskeletal system. Patients with PsA frequently have comorbidities including premature cardiovascular disease, metabolic syndrome, inflammatory bowel disease, liver disease, osteoporosis, malignancy, and ophthalmic disease. Among patients with psoriasis, the prevalence of biochemical liver abnormalities has been reported as $24-36 \%{ }^{1,2,3}$. The most common factor associated with liver abnormalities in psoriatic disease is nonalcoholic fatty liver disease (NAFLD) ${ }^{2}$. The prevalence of NAFLD in patients with psoriasis (17.4-59\%) is twice that of the general population $(7.9-28 \%)^{4,5,6,7}$. The reported risk factors associated with NAFLD in patients with psoriasis include obesity, metabolic syndrome, duration of psoriasis, PsA, severity of psoriasis or PsA, and level of high-sensitivity C-reactive protein (hsCRP) ${ }^{4,5,6,7}$. Further, patients with psoriasis had more severe NAFLD than non-psoriatic NAFLD patients ${ }^{4,7}$.

Liver abnormalities can result from the medications used to treat PsA [e.g., methotrexate (MTX) and leflunomide (LEF)]. Fifteen to thirty-five percent of patients with PsA initiating disease-modifying antirheumatic drugs (DMARD) developed biochemical liver abnormalities, which were 2.76- to 4-fold more likely in patients with PsA compared

Personal non-commercial use only. The Journal of Rheumatology Copyright $\subset$ 2020. All rights reserved. 
to patients with rheumatoid arthritis $(\mathrm{RA})^{8,9}$. In addition, the hepatotoxicity of tumor necrosis factor inhibitors (TNFi) is still debated. However, a previous study showed TNFi had a protective effect against the development of liver fibrosis in patients with $\mathrm{PsA}^{10}$. Among the liver comorbidities in patients with psoriasis, viral hepatitis is of particular interest because it is a relative contraindication for biologic therapies ${ }^{11}$.

Our aims were to determine the prevalence and incidence of liver abnormalities among patients with PsA, and to identify the factors associated with liver abnormalities in those patients.

\section{MATERIALS AND METHODS}

Study design. We used a cohort study to define the prevalence of liver abnormalities followed by a nested case-control study.

Setting. The study was conducted at the University of Toronto Psoriatic Arthritis Clinic, where patients have been followed prospectively since 1978. Other forms of inflammatory arthritis are ruled out at the initial evaluation to the PsA clinic ${ }^{12}$. The majority (98\%) of the patients fulfill the ClASsification for Psoriatic ARthritis (CASPAR) criteria for PsA ${ }^{13,14}$. Patients are assessed at 6- to 12-month intervals according to the standard protocol that consists of a detailed history, physical examination, and laboratory evaluation. Liver enzymes are measured and recorded at every protocol visit. All data are entered into a database.

Patient selection. Cases were selected from the PsA program database and included patients who had either liver biochemical abnormalities or liver disease documented after the first evaluation at the clinic. Control subjects were selected from the same database and included patients who never had any liver test abnormality or any liver disease during the followup. For the matched case-control study, each case was matched to each control by sex, age at the first evaluation in the clinic ( $\pm 5 \mathrm{yrs})$, and followup duration [time from the first evaluation to the first appearance of liver abnormalities for the cases, and the duration from the first evaluation to an evaluation date of equivalent duration ( $\pm 2 \mathrm{yrs}$ ) for controls] to identify factors at the onset of the first appearance of liver test abnormality that were associated with liver abnormalities.

Liver abnormalities. Liver biochemical abnormalities were defined by elevated transaminases $\geq 1.5$ times the upper limit of normal [ULN; aspartate transaminase (AST) $\geq 56 \mathrm{IU} / 1$ and/or alanine transaminase (ALT) $\geq 74 \mathrm{IU} / 1$ and/or alkaline phosphatase (ALP) $\geq 197 \mathrm{IU} / 1$ in males; AST $\geq 47 \mathrm{IU} / 1$ and/or ALT $\geq 66 \mathrm{IU} / 1$ and/or ALP $\geq 209 \mathrm{IU} / 1$ in females). Liver diseases included drug-induced liver injury, fatty liver, viral hepatitis, autoimmune liver disease, alcoholic liver disease, liver fibrosis, and cirrhosis. The diagnosis of liver disease was confirmed by a gastroenterologist and/ or was based on an imaging study (liver ultrasound, fibroscan, computed tomography scan, magnetic resonance imaging), viral and autoimmune serology, and histological findings on liver biopsy. The diagnosis of drug-induced liver injury was based on the presence of potential drug taken at least 1 visit prior to detection of liver abnormality, with the reasonable exclusion of other etiologies and resolution of those abnormalities after the potential drug discontinuation. Diagnosis of viral hepatitis was based on the presence of hepatitis A IgM antibody, hepatitis C antibody, hepatitis C virus RNA detection, hepatitis B surface antigen, hepatitis B core IgM antibody, or hepatitis B virus DNA detection. Diagnosis of autoimmune hepatitis was based on the presence of antinuclear antibody (ANA), anti-smooth muscle antibody, anti-liver kidney microsomal antibody type $1, \mathrm{IgG}$, absence of viral hepatitis, or abnormal liver histology ${ }^{15}$. NAFLD was defined by evidence of hepatic steatosis, either by imaging or by histology when there was no other explanation such as significant alcohol consumption, medication, or hereditary disorders. We defined alcohol-induced hepatitis by history of significant longstanding alcohol intake during the time that abnormal transaminases were detected.
Data collection. Demographic data were collected at the patients' first evaluation at the clinic including sex, ethnicity, year of entry, age, disease duration, and comorbidities [hypertension (HTN), diabetes, hyperlipidemia, body mass index (BMI)], as well as sociodemographic variables including employment status and alcohol use. Disease activity was determined by psoriasis severity [Psoriasis Area and Severity Index (PASI), score 0-72] ${ }^{16}$, swollen joint count (SJC), and tender joint count (TJC). Severity was defined by the total number of clinically damaged joints. Past and current medications, including nonsteroidal antiinflammatory drugs (NSAID), DMARD, and biologic agents were also included. Laboratory data included erythrocyte sedimentation rate (ESR), hsCRP, CRP, and serology. Seropositivity for ANA was defined as a titer of $\geq 1: 80$, and seropositivity for rheumatoid factor was defined as a level $>40 \mathrm{IU} / \mathrm{ml}$. Elevation of hsCRP and CRP were defined as a level $>1$ and $3 \mathrm{mg} / 1$, respectively. Elevation of ESR was $>13$ $\mathrm{mm} / \mathrm{h}$ in males and $>20 \mathrm{~mm} / \mathrm{h}$ in females. To identify the factors associated with liver abnormalities, these variables were also collected at the onset of the first appearance of liver test abnormality in the matched case-control study.

Statistical analysis. Descriptive statistics were presented by mean $\pm \mathrm{SD}$ and count $(\%)$. Differences among groups for continuous variables were tested using $t$ test/Wilcoxon rank-sum test, and discrete variables were tested using chi-square statistics. Normality assessment was done using the Shapiro-Wilk test. In the matched case-control analysis, the factors associated with liver abnormalities were evaluated at the first appearance of liver test abnormality. We performed a generalized estimating equation analysis A conditional univariate logistic regression was first performed on each of the variables as a predictor of the binary outcome (case vs control). All variables with a $p$ value $<0.3$ in the univariable regression analysis were selected for the multivariable regression analysis. Subsequently, backward stepwise elimination was performed to obtain a reduced model. The statistically significant difference for consideration is $p<0.05$. SAS (version 9.3; SAS Institute Inc.) was used for all statistical analyses.

Ethical review and approval were obtained from the University Health Network Research Ethics Board (08-0630). Informed consent was collected from all patients at enrollment.

\section{RESULTS}

The prevalence and the incidence of liver abnormalities in patients with PsA. Among the 1061 patients followed in the PsA clinic, 343 had liver abnormalities, providing a prevalence of liver abnormalities of $32 \%$. Of those 343 patients with liver abnormalities, 256 developed those abnormalities after their first evaluation at the clinic. The incidence of liver abnormalities was 39/1000 patient-years (PY). A total of 718 patients who had liver enzymes within the normal range and without any liver disease during followup were identified as controls.

Patient characteristics. Characteristics of PsA cases and controls at their first evaluation at the clinic are shown in Table 1 . Most of the patients $(85 \%)$ were white. There was no difference between the 2 groups with regard to sex, age at diagnosis of psoriasis or PsA, or duration of psoriasis or PsA at first visit. Daily alcohol intake was significantly higher in cases compared to controls. More cases were employed compared to controls. SJC, TJC, and use of NSAID were significantly higher in cases compared to controls, and more cases had HTN compared to controls.

Liver abnormalities in patients with PsA. Among 256 patients with PsA, liver abnormalities occurred after $8.3 \pm$ 
Table 1. Characteristics and disease-related features and treatment profiles of psoriatic arthritis (PsA) case and control populations at the first evaluation at the clinic.

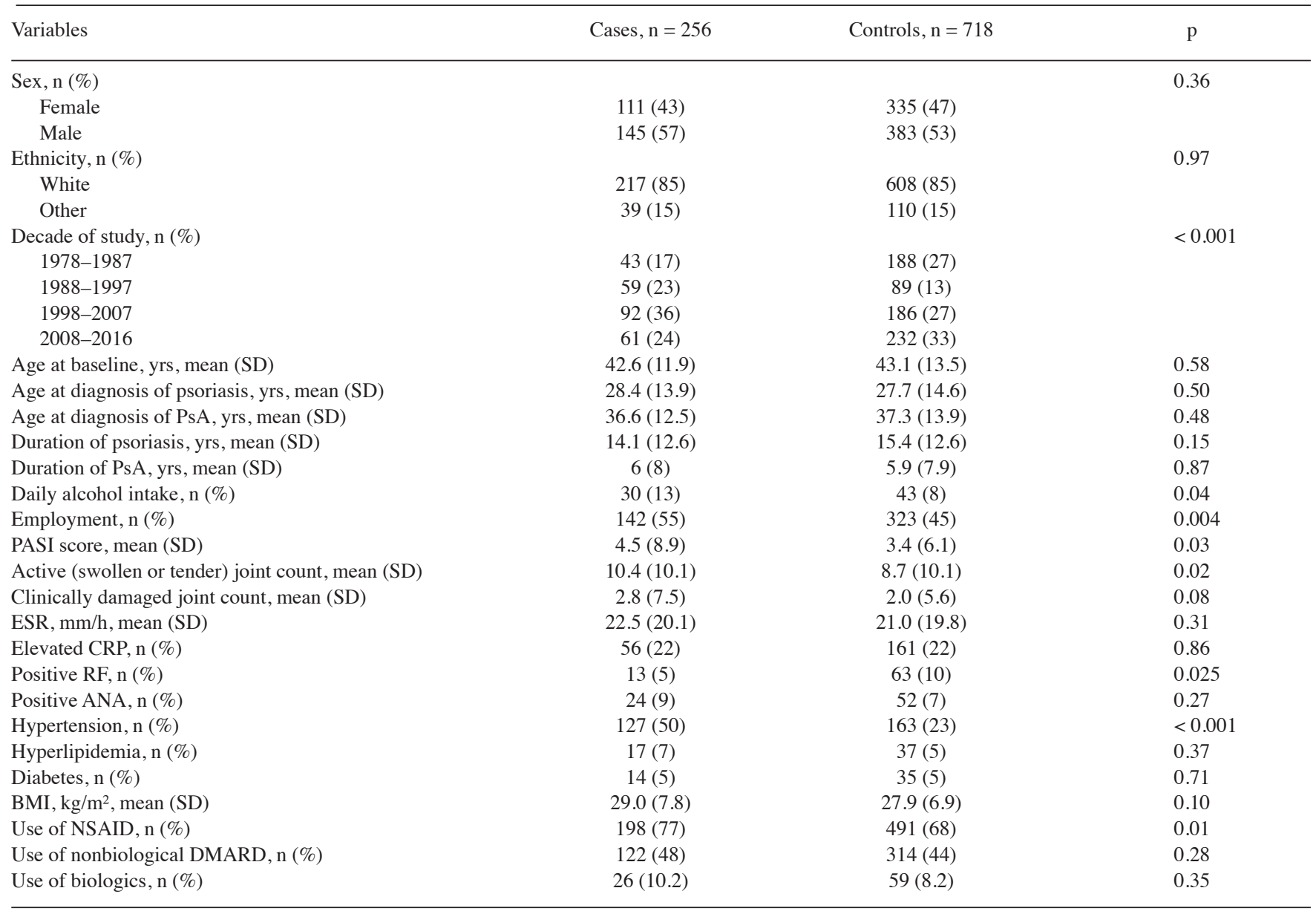

PASI: Psoriasis Area and Severity Index; ESR: erythrocyte sedimentation rate; CRP: C-reactive protein; RF: rheumatoid factor; ANA: antinuclear antibodies; BMI: body mass index; NSAID: nonsteroidal antiinflammatory drugs; DMARD: disease-modifying antirheumatic drugs.

7.8 years of followup. The mean age of patients with PsA at the onset of liver abnormalities was $50.5 \pm 12.8$ years. Eleven percent of cases took alcohol daily. The durations of psoriasis and PsA at the onset of liver abnormalities were $21.9 \pm 13.9$ and $13.8 \pm 10.9$ years, respectively. The mean PASI score, active joint count, and clinically damaged joint count were $4.0 \pm 6.4,5.4 \pm 9.7$, and $6.9 \pm 12$, respectively. The average BMI was $29.7 \pm 7.8 \mathrm{~kg} / \mathrm{m}^{2}$, and HTN was found in $52 \%$ of cases. One-half of cases used NSAID $(47 \%)$ or DMARD $(51 \%)$ at the onset of liver abnormalities. Only 29\% of cases had an identified cause of liver abnormalities or liver disease. The most common causes of liver abnormalities were drug-induced hepatitis (14\%) and fatty liver (13\%). Only 2 cases were reported to have cirrhosis. Drug-induced hepatitis occurred in 36/256 patients (MTX 18 cases, LEF 7 cases, sulfasalazine 1 case, diclofenac 2 cases, naproxen 2 cases, ibuprofen 1 case, indomethacin 1 case, celecoxib 1 case, adalimumab 1 case, acetaminophen 1 case, pravastatin 1 case). Five cases (2\%) of viral hepatitis were reported: hepatitis A (1 case), hepatitis B (1 case), hepatitis C (1 case), and Epstein-Barr virus (2 cases). Alcohol-induced hepatitis was reported in 10/256 patients (4\%). Further investigations were done only in 20 cases with liver biochemical abnormalities. The findings on liver fibroscan (12 cases) showed grade 0 (3 cases), grade 1 (6 cases), and grade 3 (3 cases). The liver ultrasound findings revealed fatty liver in 18 cases. Liver biopsy was performed in 5 cases and showed fatty liver in 4 cases and fibrosis in 1 case. Most patients (151 cases) had only 1 visit with liver biochemical abnormalities (transient abnormalities), while 105 cases had persistent or recurrent liver biochemical abnormalities. PsA patients with transient liver biochemical abnormalities had less evidence of liver disease $(23 \%$ vs $38 \%, \mathrm{p}<0.001)$ and fatty liver $(5 \%$ vs $20 \%$, $\mathrm{p}<0.001)$ compared to PsA patients with persistent or recurrent liver biochemical abnormalities.

Factors associated with liver abnormalities in PsA patients. To determine the factors at the first appearance of liver test

Personal non-commercial use only. The Journal of Rheumatology Copyright $\subset$ 2020. All rights reserved. 
abnormalities that contributed to liver abnormalities, we matched 204 cases to 204 controls on age, sex, and duration of followup. Characteristics and disease-associated features at the onset of liver abnormalities in the matched case-control study are shown in Table 2. Durations of psoriasis and PsA were significantly longer in cases compared to controls. Cases had significantly higher clinically damaged joint count and ESR compared to controls. HTN, hyperlipidemia, and higher BMI were significantly more prevalent in cases. The use of NSAID was significantly less common in the cases; however, TNFi were more commonly used in cases than in controls.

In univariate analysis, the variables associated with liver abnormalities were employment (OR $0.34,95 \% \mathrm{CI}$ $0.22-0.55, \mathrm{p}<0.0001$ ), clinically damaged joint count (OR $1.05,95 \%$ CI $1.02-1.07, \mathrm{p}=0.0001)$, duration of psoriasis (OR 1.02, 95\% CI 1.01-1.04, $\mathrm{p}=0.005$ ), duration of PsA (OR 1.08, 95\% CI 1.05-1.11, p < 0.0001), ESR (OR 1.02, 95\% CI 1.00-1.03, p = 0.03), HTN (OR 3.33, $95 \%$ CI 2.05-5.43, p < 0.0001), hyperlipidemia (OR 2.50, $95 \%$ CI $1.45-4.32, \mathrm{p}=0.001)$, BMI (OR $1.04,95 \%$ CI $1.00-1.09, \mathrm{p}=0.04)$, use of TNFi(OR $1.67,95 \%$ CI $1.08-2.57$, $\mathrm{p}=0.02$; Table 3 ). However, in the multivariate analysis, these were independent factors associated with liver abnormalities in patients with PsA after adjusting for other covariates: BMI (OR 1.07, 95\% CI 1.02-1.12, $\mathrm{p}=0.007$ ), daily alcohol intake (OR 4.46, 95\% CI 1.30-15.28, $\mathrm{p}=0.02$ ), damaged joint count (OR 1.04, 95\% CI 1.01-1.08, $\mathrm{p}=0.01$ ), elevated CRP (OR 2.00, 95\% CI 1.04-3.85, $\mathrm{p}=0.04$ ), use of MTX/LEF (OR 4.39, 95\% CI 1.67-11.54, $\mathrm{p}=0.003$ ), and use of TNFi (OR 10.56, 95\% CI 3.63-30.69, p < 0.0001).

\section{DISCUSSION}

In our large longitudinal cohort study, we found the prevalence and the incidence of liver abnormalities were 0.32 and $0.039 \mathrm{PY}$ of followup, respectively. The prevalence is comparable with previous studies among patients with psoriasis of $24-36 \% \%^{1,2}$. The common causes of liver abnormalities in our cohort were drug-induced hepatitis and fatty liver disease, which were in agreement with the findings observed in a psoriasis study ${ }^{2}$. Higher prevalence of liver abnormalities in patients with PsA could be related to more widespread use of hepatotoxic agents such as MTX or LEF. Candia, et $a l$ found that the risk of NAFLD was significantly higher in psoriasis patients with PsA (OR 2.25, 95\% CI 1.37-3.71) Ogdie, et ${ }^{17}{ }^{17}$ made a similar observation, noting that the risk for liver disease was higher among patients with PsA than patients with psoriasis alone or RA. In our study, most liver biochemical abnormalities were transient and rarely led to liver fibrosis or cirrhosis.

We demonstrated an association between higher BMI and liver abnormalities in patients with PsA. This finding was noted by Rahmioglu, et al, who showed that the variation in the liver biochemical abnormalities was associated with BMI in both males and females in a twin pair cohort study that included 5380 subjects $^{18}$. These results could be explained by the relationship between BMI and NAFLD. Chang, et al demonstrated an increasing baseline BMI had a strong linear relationship with NAFLD among a cohort study of 77,425 metabolically healthy obese individuals who were followed for 4.5 years and NAFLD determined by ultrasound ${ }^{19}$. An observational cohort study of the incidence of moderate liver toxicity ( $\geq 1.5 \times \mathrm{ULN})$ of MTX in the management

Table 2. Characteristics and disease-related features and treatment profiles of the cases and their matched controls (204 pairs) at the onset of liver abnormalities.

\begin{tabular}{|c|c|c|c|}
\hline Variables & Cases, $\mathrm{n}=204$ & Controls, $\mathrm{n}=204$ & $\mathrm{p}$ \\
\hline Whites, n (\%) & $168(82)$ & $168(82)$ & 1.00 \\
\hline Duration of psoriasis, yrs, mean (SD) & $25.1(14.7)$ & $21.3(13.8)$ & 0.009 \\
\hline Daily alcohol intake, $\mathrm{n}(\%)$ & $19(10)$ & $12(6)$ & 0.14 \\
\hline Employment, $\mathrm{n}(\%)$ & $89(44)$ & $135(66)$ & $<0.001$ \\
\hline PASI score, mean (SD) & $4.0(7.3)$ & $3.5(5.3)$ & 0.40 \\
\hline $\mathrm{ESR}, \mathrm{mm} / \mathrm{h}$, mean $(\mathrm{SD})$ & $16.9(17.4)$ & $13.8(12.4)$ & 0.04 \\
\hline Elevated CRP, n (\%) & $77(38)$ & $67(33)$ & 0.30 \\
\hline Positive RF, n (\%) & $6(3)$ & $3(2)$ & 0.36 \\
\hline Positive ANA, n (\%) & $31(15)$ & $27(13)$ & 0.57 \\
\hline Hypertension, $\mathrm{n}(\%)$ & $115(56)$ & $66(32)$ & $<0.001$ \\
\hline Hyperlipidemia, n (\%) & $51(26)$ & $25(12)$ & $<0.001$ \\
\hline Use of TNFi, $\mathrm{n}(\%)$ & $92(45)$ & $70(37)$ & 0.03 \\
\hline
\end{tabular}

PsA: psoriatic arthritis; PASI: Psoriasis Area and Severity Index; ESR: erythrocyte sedimentation rate; CRP: C-reactive protein; RF: rheumatoid factor; ANA: antinuclear antibodies; BMI: body mass index; NSAID: nonsteroidal antiinflammatory drugs; DMARD: disease-modifying antirheumatic drugs; TNFi: tumor necrosis factor inhibitors. 
Table 3. Univariate analysis of factors associated with liver abnormalities in the matched case-control study in the PsA cohort.

\begin{tabular}{lcc}
\hline Variables & OR $(95 \%$ CI $)$ & $\mathrm{p}$ \\
\hline Duration of psoriasis, yrs & $1.02(1.01-1.04)$ & 0.005 \\
Duration of PsA, yrs & $1.08(1.05-1.11)$ & $<0.0001$ \\
Daily alcohol intake & $1.70(0.78-3.71)$ & 0.18 \\
Employment & $0.34(0.22-0.55)$ & $<0.0001$ \\
PASI score & $1.01(0.98-1.04)$ & 0.41 \\
Active (swollen or tender) joint count & $1.03(0.99-1.06)$ & 0.06 \\
Clinically damaged joint count & $1.05(1.02-1.07)$ & 0.0001 \\
ESR & $1.02(1.00-1.03)$ & 0.03 \\
Elevated CRP & $1.27(0.83-1.95)$ & 0.28 \\
Positive RF & $2.50(0.49-12.89)$ & 0.27 \\
Positive ANA & $1.18(0.67-2.09)$ & 0.56 \\
Hypertension & $3.33(2.05-5.43)$ & $<0.0001$ \\
Hyperlipidemia & $2.50(1.45-4.32)$ & 0.001 \\
Diabetes & $1.64(0.85-3.19)$ & 0.14 \\
BMI & $1.04(1.00-1.09)$ & 0.04 \\
Use of NSAID & $0.63(0.40-0.99)$ & 0.05 \\
Use of MTX/LEF & $1.28(0.86-1.91)$ & 0.23 \\
Use of TNFi & $1.67(1.08-2.57)$ & 0.02 \\
\hline & & \\
\hline
\end{tabular}

PsA: psoriatic arthritis; PASI: Psoriasis Area and Severity Index; ESR: erythrocyte sedimentation rate; CRP: C-reactive protein; RF: rheumatoid factor; ANA: antinuclear antibodies; BMI: body mass index; NSAID: nonsteroidal antiinflammatory drugs; MTX: methotrexate; LEF: leflunomide; TNFi: tumor necrosis factor inhibitors.

of rheumatic disease also identified BMI $\left(\geq 30 \mathrm{~kg} / \mathrm{m}^{2}\right)$ as a predictor of liver biochemical abnormalities ${ }^{20}$. A systematic review conducted by Montaudie, et al, revealed that obesity was associated with a significantly increased risk of liver fibrosis in patients with psoriasis receiving $\mathrm{MTX}^{21}$.

Alcohol consumption contributes to a variety of liver diseases, either by causing alcoholic liver disease or as the aggravating factor in chronic viral hepatitis, NALFD, and drug-induced hepatitis. We found that patients with PsA who consumed alcohol daily were more likely to have liver abnormalities than those who did not or were occasional alcohol consumers. Among patients with RA receiving MTX or LEF, daily alcohol use (1-2 drinks per day) was associated with ALT/AST elevation compared to none/ occasional use (OR 1.97, 95\% CI 1.18-3.28) ${ }^{7}$. Malatjalian, et al demonstrated that occasional alcohol consumption ( $\leq 3$ drinks per week) was not associated with increased risk of MTX hepatotoxicity in patients with psoriasis ${ }^{22}$. Humphreys, et al showed that among 11,839 patients with RA taking MTX and weekly alcohol consumption of $<14$ units per week, there was no increased risk of transaminitis $^{23}$. However, more than 21 units per week of alcoholic drinking was associated with a significant risk of transaminitis. This result was supported by the study of Laharie, et $a l^{24}$, which found that high alcohol consumption (>14 units per week) was associated with severe liver fibrosis among patients diagnosed with chronic inflammatory disorders who were receiving MTX.
The chronic inflammatory process from psoriatic disease itself was also associated with the increased prevalence of liver damage, NAFLD, and liver fibrosis compared with non-psoriatic patients or the general population. Significant factors associated with NAFLD in psoriatic patients include longer duration of psoriatic disease and greater severity of psoriasis assessed by PASI score ${ }^{3,4,5,6}$. Patients with PsA who achieved minimal disease activity after receiving TNFi for 12 months exhibited lower incidence of worsening liver steatosis compared with PsA patients with persistently active disease $^{25}$. We found that a higher damaged joint count and elevated CRP level were associated with liver abnormalities. Associations between high BMI and joint damage and the presence of liver abnormalities may be mediated through TNF- $\alpha$ and insulin resistance. TNF- $\alpha$ is found in skin and synovial tissue and TNF- $\alpha$ levels have been elevated in active, severe psoriatic disease ${ }^{26,27}$. Moreover, TNF- $\alpha$ mediates matrix metalloproteinases that lead to cartilage and joint destruction ${ }^{28}$. TNF- $\alpha$ promotes insulin resistance and induces transforming growth factor $\beta$ and connective growth factor, which have been implicated in the development of NAFLD and contribute to hepatic fibrosis ${ }^{29,30}$. In addition, an elevation of hsCRP, a marker of chronic inflammation, has been reported as the predictor of NAFLD ${ }^{31}$. This concept is supported by the report by Campanati, et al, who found that moderate to severe psoriasis patients with NAFLD receiving etanercept had significant reduction in the risk of the development of hepatic fibrosis compared with patients receiving psoralen and UVA therapy ${ }^{32}$.

We found that patients with PsA who were receiving MTX or LEF were more likely to have liver abnormalities than patients who were not taking these medications. This is not surprising because both are associated with hepatotoxicity. Previous studies showed that $14.5-35 \%$ of patients with PsA receiving MTX had elevated AST/ALT levels and patients with PsA had a 2.7- to 4-fold greater likelihood of these abnormalities compared to patients with $\mathrm{RA}^{8,9}$. Further, PsA patients with high cumulative dose of MTX had a prevalence of liver fibrosis of $22 \%$, which related to the number of risk factors that contributed to liver fibrosis or NAFLD (excessive alcohol consumption, diabetes, obesity, renal impairment) ${ }^{33}$. Higher incidence of elevated ALT level were found in patients with PsA receiving LEF (12.5\%) compared to placebo $(5.4 \%)$ in a multinational, double-blind, randomized, placebo-controlled clinical trial. Only a few patients were withdrawn from the study because of ALT levels above 3 times the ULN and no cases of severe liver toxicity were reported in LEF-treated patients ${ }^{34}$.

The hepatotoxicity of TNFi is still inconclusive. Our study showed TNFi are associated with the incidence of liver abnormalities in patients with PsA. However, it should be noted that the majority of the patients had had their conventional DMARD prior to the anti-TNF agents, and it is possible that in patients with liver disease, the anti-TNF

Personal non-commercial use only. The Journal of Rheumatology Copyright $(\subset) 2020$. All rights reserved. 
agents were used instead of conventional DMARD to avoid worsening of liver disease. Randomized clinical trials with anti-TNF agents reported few discontinuations due to increased ALT/AST ${ }^{35,36,37,38,39}$. Nonetheless, several cases of psoriasis with autoimmune hepatitis (AIH) triggered by TNFi were reported in the literature ${ }^{40}$. Two of the TNFi observed in the literature were infliximab and adalimumab, and the onset of liver damage occurred between 4 and 26 weeks. All patients discontinued TNFi and were treated with glucocorticosteroids ( \pm azathioprine). ANA was expressed in all patients and liver abnormalities were resolved within 3 months after treatment. However, our study did not show the association between positivity of ANA and liver abnormalities, and no AIH was reported.

Although we studied a large number of patients with PsA in our cohort, we could identify an etiology for the liver biochemical abnormalities in only $30 \%$. NAFLD was identified in only $13 \%$ of PsA cases, which is less than in the previous studies of psoriasis ${ }^{4,5,6,7}$.

Liver abnormalities are commonly found in patients with PsA. We found that higher BMI, daily alcohol intake, higher damaged joint count, elevated CRP, and use of MTX/ LEF or TNFi are associated with the development of liver abnormalities in patients with PsA. We recommend monitoring liver function tests in these high-risk patients. This is important in the management of patients with PsA because many of the therapeutic options may aggravate or even lead to liver abnormalities in this patient population.

\section{REFERENCES}

1. Finet A, Viguier M, Chazouilleres O, Amatore F, Paul C, Richard MA, et al; Groupe de Recherche sur le Psoriasis de la Société Française de Dermatologie. Liver test abnormalities in patients admitted for severe psoriasis: prevalence and associated risk factors. J Eur Acad Dermatol Venereol 2016;30:1742-8.

2. Tula E, Ergun T, Seckin D, Ozgen Z, Avsar E. Psoriasis and the liver: problems, causes and course. Australas J Dermatol 2017;58:194-9.

3. Fiore M, Leone S, Maraolo AE, Berti E, Damiani G. Liver illness and psoriatic patients. Biomed Res Int 2018;2018:3140983.

4. Miele L, Vallone S, Cefalo C, La Torre G, Di Stasi C, Vecchio FM, et al. Prevalence, characteristics and severity of non-alcoholic fatty liver disease in patients with chronic plaque psoriasis. J Hepatol 2009;51:778-86.

5. Gisondi P, Targher G, Zoppini G, Girolomoni G. Non-alcoholic fatty liver disease in patients with chronic plaque psoriasis. J Hepatol 2009;51:758-64.

6. Candia R, Ruiz A, Torres-Robles R, Chavez-Tapia N, Mendez-Sanchez N, Arrese M. Risk of non-alcoholic fatty liver disease in patients with psoriasis: a systematic review and meta-analysis. J Eur Acad Dermatol Venereol 2015;29:656-62.

7. Madanagobalane S, Anandan S. The increased prevalence of non-alcoholic fatty liver disease in psoriatic patients: a study from South India. Australas J Dermatol 2012;53:190-7.

8. Curtis JR, Beukelman T, Onofrei A, Cassell S, Greenberg JD, Kavanaugh A, et al. Elevated liver enzyme tests among patients with rheumatoid arthritis or psoriatic arthritis treated with methotrexate and/or leflunomide. Ann Rheum Dis 2010;69:43-7.

9. Tilling L, Townsend S, David J. Methotrexate and hepatic toxicity in rheumatoid arthritis and psoriatic arthritis. Clin Drug Investig 2006;26:55-62.

10. Seitz M, Reichenbach S, Moller B, Zwahlen M, Villiger PM, Dufour JF. Hepatoprotective effect of tumour necrosis factor alpha blockade in psoriatic arthritis: a cross-sectional study. Ann Rheum Dis 2010;69:1148-50.

11. Damiani G, Franchi C, Pigatto P, Altomare A, Pacifico A, Petrou S, et al. Outcomes assessment of hepatitis $\mathrm{C}$ virus-positive psoriatic patients treated using pegylated interferon in combination with ribavirin compared to new Direct-Acting Antiviral agents. World J Hepatol 2018;10:329-36.

12. Gladman DD, Shuckett R, Russell ML, Thorne JC, Schachter RK. Psoriatic arthritis (PSA)--an analysis of 220 patients. Q J Med 1987;62:127-41.

13. Taylor W, Gladman D, Helliwell P, Marchesoni A, Mease P, Mielants H; CASPAR Study Group. Classification criteria for psoriatic arthritis: development of new criteria from a large international study. Arthritis Rheum 2006;54:2665-73.

14. Chandran V, Schentag CT, Gladman DD. Sensitivity of the classification of psoriatic arthritis criteria in early psoriatic arthritis. Arthritis Rheum 2007;57:1560-3.

15. Hennes EM, Zeniya M, Czaja AJ, Par'es A, Dalekos GN, Krawitt EL, et al. Simplified criteria for the diagnosis of autoimmune hepatitis. Hepatology 2008;48:169-76.

16. Marks R, Barton SP, Shuttleworth D, Finlay AY. Assessment of disease progress in psoriasis. Arch Dermatol 1989;125:235-40.

17. Ogdie A, Grewal SK, Noe MH, Shin DB, Takeshita J, Chiesa Fuxench ZC, et al. Risk of incident liver disease in patients with psoriasis, psoriatic arthritis, and rheumatoid arthritis: a population-based study. J Invest Derm 2018;138:760-7.

18. Rahmioglu N, Andrew T, Cherkas L, Surdulescu G, Swaminathan $\mathrm{R}$, Spector T, et al. Epidemiology and genetic epidemiology of the liver function test proteins. PLoS One 2009;4:e4435.

19. Chang Y, Jung HS, Cho J, Zhang Y, Yun KE, Lazo M, et al. Metabolically healthy obesity and the development of nonalcoholic fatty liver disease. Am J Gastroenterol 2016;111:1133-40.

20. Schmajuk G, Miao Y, Yazdany J, Boscardin WJ, Daikh DI, Steinman MA. Identification of risk factors for elevated transaminases in methotrexate users through an electronic health record. Arthritis Care Res 2014;66:1159-66.

21. Montaudie H, Sbidian E, Paul C, Maza A, Gallini A, Aractingi S, et al. Methotrexate in psoriasis: a systematic review of treatment modalities, incidence, risk factors and monitoring of liver toxicity. J Eur Acad Dermatol Venereol 2011;25 Suppl 2:12-8.

22. Malatjalian DA, Ross JB, Williams CN, Colwell SJ, Eastwood BJ Methotrexate hepatotoxicity in psoriatics: report of 104 patients from Nova Scotia, with analysis of risks from obesity, diabetes and alcohol consumption during long term follow-up. Can J Gastroenterol 1996;10:369-75.

23. Humphreys JH, Warner A, Costello R, Lunt M, Verstappen SM, Dixon WG. Quantifying the hepatotoxic risk of alcohol consumption in patients with rheumatoid arthritis taking methotrexate. Ann Rheum Dis Online 2017;76:1509-14.

24. Laharie D, Seneschal J, Schaeverbeke T, Doutre MS, Longy-Boursier M, Pellegrin JL, et al. Assessment of liver fibrosis with transient elastography and FibroTest in patients treated with methotrexate for chronic inflammatory diseases: a case-control study. J Hepatol 2010;53:1035-40.

25. Di Minno MN, Iervolino S, Peluso R, Russolillo A, Lupoli R, Scarpa R, et al; CaRRDS Study Group. Hepatic steatosis and disease activity in subjects with psoriatic arthritis receiving tumor necrosis factor-alpha blockers. J Rheumatol 2012;39:1042-6.

26. Ritchlin C, Haas-Smith SA, Hicks D, Cappuccio J, Osterland CK, Looney RJ. Patterns of cytokine production in psoriatic synovium. J Rheumatol 1998;25:1544-52. 
27. Balding J, Kane D, Livingstone W, Mynett-Johnson L, Bresnihan B, Smith O, et al. Cytokine gene polymorphisms: association with psoriatic arthritis susceptibility and severity. Arthritis Rheum 2003;48:1408-13.

28. Fraser A, Fearon U, Billinghurst RC, Ionescu M, Reece R, Barwick $\mathrm{T}$, et al. Turnover of type II collagen and aggrecan in cartilage matrix at the onset of inflammatory arthritis in humans: relationship to mediators of systemic and local inflammation. Arthritis Rheum 2003;48:3085-95.

29. Breitkopf K, Sawitza I, Gressner AM. Characterization of intracellular pathways leading to coinduction of thrombospondin-1 and TGF-beta1 expression in rat hepatic stellate cells. Growth Factors 2005;23:77-85.

30. Gressner OA, Gressner AM. Connective tissue growth factor: a fibrogenic master switch in fibrotic liver diseases. Liver Int 2008;28:1065-79.

31. Lee J, Yoon K, Ryu S, Chang Y, Kim HR. High-normal levels of hs-CRP predict the development of non-alcoholic fatty liver in healthy men. PLoS One 2017;12:e0172666.

32. Campanati A, Ganzetti G, Di Sario A, Damiani A, Sandroni L, Rosa $\mathrm{L}$, et al. The effect of etanercept on hepatic fibrosis risk in patients with non-alcoholic fatty liver disease, metabolic syndrome, and psoriasis. J Gastroenterol 2013;48:839-46.

33. Lindsay K, Fraser AD, Layton A, Goodfield M, Gruss H, Gough A. Liver fibrosis in patients with psoriasis and psoriatic arthritis on long-term, high cumulative dose methotrexate therapy. Rheumatology 2009;48:569-72.

34. Kaltwasser JP, Nash P, Gladman D, Rosen CF, Behrens F, Jones P, et al; Treatment of Psoriatic Arthritis Study Group. Efficacy and safety of leflunomide in the treatment of psoriatic arthritis and psoriasis: a multinational, double-blind, randomized, placebo-controlled clinical trial. Arthritis Rheum 2004;50:1939-50.

35. Fenix-Caballero S, Alegre-del Rey EJ, Castano-Lara R, Puigventos-Latorre F, Borrero-Rubio JM, Lopez-Vallejo JF. Direct and indirect comparison of the efficacy and safety of adalimumab, etanercept, infliximab and golimumab in psoriatic arthritis. J Clin Pharm Ther 2013;38:286-93.

36. Kavanaugh A, McInnes I, Mease P, Krueger GG, Gladman D, Gomez-Reino J, et al. Golimumab, a new human tumor necrosis factor alpha antibody, administered every four weeks as a subcutaneous injection in psoriatic arthritis: twenty-four-week efficacy and safety results of a randomized, placebo-controlled study. Arthritis Rheum 2009;60:976-86.

37. Kavanaugh A, McInnes IB, Mease P, Krueger GG, Gladman D, van der Heijde D, et al. Clinical efficacy, radiographic and safety findings through 5 years of subcutaneous golimumab treatment in patients with active psoriatic arthritis: results from a long-term extension of a randomised, placebo-controlled trial (the GO-REVEAL study). Ann Rheum Dis 2014;73:1689-94.

38. Mease PJ, Kivitz AJ, Burch FX, Siegel EL, Cohen SB, Ory P, et al. Etanercept treatment of psoriatic arthritis: safety, efficacy, and effect on disease progression. Arthritis Rheum 2004;50:2264-72.

39. Paramarta JE, De Rycke L, Heijda TF, Ambarus CA, Vos K, Dinant HJ, et al. Efficacy and safety of adalimumab for the treatment of peripheral arthritis in spondyloarthritis patients without ankylosing spondylitis or psoriatic arthritis. Ann Rheum Dis 2013;72:1793-9.

40. Nakayama S. Autoimmune hepatitis triggered by anti-TNF-alpha therapy. Case Rep Med 2013;2013:561748. 